\title{
PRIVATIZAÇÃO NUM MERCADO MISTO COM DECISÕES SEQUENCIAIS
}

\author{
Fernanda A. Ferreira
}

ESEIG.IPP, Unidade de Investigação Aplicada em Gestão (UNIAG), Instituto Politécnico do Porto

Rua D. Sancho I, 981, 4480-876 Vila do Conde, Portugal

fernandaamelia@eseig.ipp.pt

\section{Flávio Ferreira}

ESEIG.IPP, Unidade de Investigação Aplicada em Gestão (UNIAG), Instituto Politécnico do Porto

Rua D. Sancho I, 981, 4480-876 Vila do Conde, Portugal

flavioferreira@eseig.ipp.pt

\section{Resumo}

Consideramos um mercado no qual competem uma empresa pública e uma empresa privada, decidindo, de forma sequencial, as quantidades a produzir. O governo impõe um imposto sobre as quantidades comercializadas, de acordo com uma função que consiste numa soma ponderada entre o bem-estar público e a receita total obtida pela aplicação desse imposto. O objetivo deste trabalho é estudar o efeito da privatização da empresa pública, (i) quando a empresa líder é a empresa pública; e (ii) quando a empresa líder é a empresa privada. Além disso, fazemos uma comparação entre os resultados obtidos nos dois modelos estudados.

Palavras-Chaves: Duopólio misto; Modelo de Stackelberg; Organização Industrial; Teoria dos Jogos.

\begin{abstract}
We consider a market in which a public firm and a private firm compete by deciding, sequentially, the quantities to be produced. The government imposes a tax on the quantities, according to a function that is a weighted sum of the welfare and the total revenue obtained by applying this tax. The aim of this paper is to study the effect of privatization, (i) when the leader is the public firm; and (ii) when the leader is a private firm. Furthermore, we do a comparison between the results from the two models.
\end{abstract}

Keywords: $\quad$ Mixed duopoly; Stackelberg model; Industrial Organization; Game Theory.

\section{INTRODUÇÃO}

Neste artigo, estudamos um mercado constituído por uma empresa pública e uma empresa privada que produzem, e vendem, um mesmo artigo homogéneo, isto é, indiferenciado aos olhos dos compradores. A empresa privada decide, e torna pública, a quantidade que vai colocar no mercado, antes de a empresa pública o fazer. Assim, a empresa pública decide a quantidade que irá produzir conhecendo já a quantidade da sua concorrente. Além disso, o modelo considera que o governo fixa um imposto que incidirá sobre as quantidades comerercializadas por cada uma das empresas, e divulga a respetiva taxa antes de as empresas tomarem as decisões sobre as rerspetivas quantidades a produzir. $\mathrm{O}$ governo tem como objetivo maximizar uma média ponderada entre o bem-estar social e a receita fiscal. No modelo apresentado, avaliamos o impacto da preferência do governo pela receita fiscal na decisão de privatizar a empresa pública. 
O mesmo estudo é feito para o modelo em que a empresa líder é a empresa pública.

\section{REVISÃO DE LITERATURA}

O interesse no estudo de oligopólios mistos tem vindo a crescer nos últimos anos. Um aspeto importante nestes estudos prende-se com o efeito da privatização, em particular no bem-estar social. Chao e Yu (2006) avaliaram o impacto de privatização parcial e de competição internacional na variação da taxa ótima, tendo concluído que uma competição internacional faz baixar a taxa ótima, enquanto que uma privatização parcial a aumenta. White (1996) e Fjell e Heywood (2004) introduziram subsídios em modelos de duopólio misto. Usualmente, estes autores consideraram o bem-estar social como função objetivo, quer para o governo quer para a empresa pública. Porém, Matsumura (1998) considerou, para função objetivo, uma média ponderada entre o bem-estar social modificado e o lucro da empresa. Este bem-estar social modificado leva o governo a ter uma maior preferência pelo excedente do consumidor do que pelos lucros das empresas.

Também as taxas cobradas pelos governos constituem matéria de interesse para estudo. Brander e Spencer (1984) mostraram que a aplicação de taxas tem efeito de alteração no lucro, para além do seu efeito sobre a receita tarifária. Larue e Gervais (2002) estudaram o efeito da maximização da receita tarifária num modelo de Cournot (escolha simultânea das quantidades). Ferreira e Ferreira (2009) analisaram o efeito da maximização da receita fiscal num modelo internacional de Bertrand (escolha simultânea dos preços), com produtos diferenciados e com desconhecimento dos custos de produção da empresa concorrente. Clarke e Collie (2006) estudaram um problema análogo, mas com conhecimento mútuo dos custos de produção.

Kato (2008) considerou um duopólio misto de Cournot, assumindo que o governo tem uma maior preferência na receita total das taxas do que no bem-estar social, enquanto que a empresa pública tem interesse apenas no bem-estar social. Neste modelo, o autor estudou a relação entre privatização da empresa pública e a preferência do governo pela receita total das taxas, tendo concluído que (i) o governo fixa uma taxa mais elevada no duopólio misto do que no duopólio privatizado, e que (ii) o interesse do governo na privatização depende da sua preferência pela receita total das taxas.

Ferreira e Ferreira (2014) consideraram funções objetivo iguais às do Kato para o governo e para a empresa pública, mas em que as empresas seguem uma competição à la Stackelberg (decidem as quantidades de modo sequencial), com a empresa pública como líder, em vez de tomarem decisões simultâneas.

\section{O DUOPÓLIO MISTO: EMPRESA PRIVADA LÍDER}

Consideremos um mercado no qual competem duas empresas, uma pública e uma privada, com um produto homogéneo. As empresas têm que decidir as quantidades que colocarão no mercado, e assumimos que a empresa privada toma e anuncia a sua decisão primeiro. As empresas estão, assim, em competição à la Stackelberg. A função de demanda inversa é dada por

$$
p=a-Q
$$

onde $p$ é o preço do mercado, $Q=q_{1}+q_{2}$ é a quantidade agregada, sendo $q_{1}$ a quantidade produzida pela empresa privada $F_{1}$ e $q_{2}$ a quantidade produzida pela empresa pública $F_{2}$, e $a>0$. Ambas as empresas têm a mesma estrutura de custos de produção: $C\left(q_{i}\right)=q_{i}^{2} / 2$, com $i=1,2$.

Supomos ainda, como dissemos na Introdução, que o governo impõe um taxa $t$ de imposto para ambas as empresas, que incidirá sobre as quantidades produzidas (e colocadas no mercado).

O modelo consiste, portanto, no seguinte jogo com três etapas:

- Na primeira etapa, o governo fixa a taxa $t$ do imposto; 
- Na segunda etapa, a empresa privada $F_{1}$ decide a quantidade $q_{1}$ que irá produzir;

- Na terceira etapa, a empresa pública $F_{2}$ decide a quantidade $q_{2}$ que irá produzir. A função objetivo para a empresa privada $F_{1}$ é o seu lucro:

$$
\pi_{1}=\left(a-q_{1}-q_{2}\right) q_{1}-\frac{q_{1}^{2}}{2}-t q_{1}
$$

enquanto que a função objetivo para a empresa pública $F_{2}$ é o bem-estar social:

$$
W=\frac{\left(q_{1}+q_{2}\right)^{2}}{2}+\left(a-q_{1}-q_{2}\right)\left(q_{1}+q_{2}\right)-\frac{q_{1}^{2}+q_{2}^{2}}{2}-T,
$$

onde $T=t\left(q_{1}+q_{2}\right)$ é a receita fiscal.

A função objetivo para o governo é

$$
U=W+(1+\alpha) T
$$

onde o parâmetro $\alpha$ representa o peso da preferência do governo pela receita fiscal. Consideramos que o governo tem uma maior preferência por $T$ do que por $W$, pelo que $\alpha \geq 0$ (outra situação seria inconsistente com a realidade). Se $\alpha=0$, o governo tem igual preferência por $T$ e por $W$; Para $\alpha>0$, quanto maior o valor de $\alpha$, maior a preferência do governo por $T$.

Como é habitual em jogos dinâmicos, resolveremos o nosso problema por indução recíproca. Maximizando a função objetivo $W$ da empresa pública, obtemos

$$
q_{2}=\frac{a-q_{1}-t}{2}
$$

Utilizando agora este resultado na maximização do lucro $\pi_{1}$ da empresa privada, obtemos

$$
q_{1}=\frac{a-t}{4},
$$

e, portanto,

$$
q_{2}=\frac{3(a-t)}{8} .
$$

A função objetivo $U$ do governo pode, agora, escrever-se como:

$$
U=\frac{(a-t)(21 a+t(40 \alpha+19))}{64} .
$$

Maximizando esta função objetivo, o valor ótimo para a taxa de imposto no duopólio misto vem dado por $^{1}$

$$
t^{M}=\frac{a(20 \alpha-1)}{40 \alpha+19} .
$$

Daqui resulta que, se $\alpha>1 / 20$, a taxa ótima de imposto é positiva; e se $0 \leq \alpha<1 / 20$, a taxa ótima de imposto é negativa, o que significa que o governo subsidia as empresas.

Proposição 1. A taxa de imposto fixada pelo governo aumenta com a preferência do governo pela receita fiscal.

Das expressões acima, resulta ainda o seguinte resultado:

\footnotetext{
${ }^{1}$ Ao longo do artigo, utilizamos a notação $M$ para duopólio misto e $P$ para duopólio pós-privatização.
} 
Proposição 2. No duopólio misto, com a empresa privada como líder, os valores em equilíbrio são:

$$
\begin{gathered}
q_{1}^{M}=\frac{5 a(\alpha+1)}{40 \alpha+19}, \\
q_{2}^{M}=\frac{15 a(\alpha+1)}{2(40 \alpha+19)}, \\
Q^{M}=\frac{25 a(\alpha+1)}{2(40 \alpha+19)}, \\
p^{M}=\frac{a(55 \alpha+13)}{2(40 \alpha+19)}, \\
U^{M}=\frac{25 a^{2}(\alpha+1)^{2}}{4(40 \alpha+19)}, \\
\pi_{1}^{M}=\frac{25 a^{2}(\alpha+1)^{2}}{(40 \alpha+19)^{2}}, \\
T^{M}=\frac{25 a^{2}(\alpha+1)(20 \alpha-1)}{2(40 \alpha+19)^{2}}, \\
W^{M}=\frac{525 a^{2}(\alpha+1)^{2}}{4(40 \alpha+19)^{2}} .
\end{gathered}
$$

Observamos que as quantidades produzidas por cada uma das empresas, e portanto a quantidade agregada no mercado, diminuem à medida que aumenta a preferência do governo pela receita fiscal. Tal deve-se ao facto de o valor ótimo para a taxa de imposto estar positivamente correlacionada com o parâmetro $\alpha$.

\section{O DUOPÓLIO MISTO: EMPRESA PÚBLICA LÍDER}

Ferreira e Ferreira (2014) estudaram o problema apresentado na secção anterior, assumindo que a empresa pública $F_{2}$ era líder e a empresa privada $F_{1}$ era a empresa seguidora. Com essas hipóteses, obtiveram o seguinte resultado:

No duopólio misto, com a empresa pública como líder, os valores em equilíbrio são²:

$$
\begin{gathered}
\tilde{t}^{M}=\frac{a(8 \alpha-1)}{16 \alpha+7}, \\
\tilde{q}_{1}^{M}=\frac{12 a(\alpha+1)}{7(16 \alpha+7)}, \\
\tilde{q}_{2}^{M}=\frac{20 a(\alpha+1)}{7(16 \alpha+7)},
\end{gathered}
$$

\footnotetext{
${ }^{2}$ Utilizamos o para os resultados em que a empresa líder é a empresa pública.
} 


$$
\begin{gathered}
\tilde{Q}^{M}=\frac{32 a(\alpha+1)}{7(16 \alpha+7)}, \\
\tilde{p}^{M}=\frac{a(80 \alpha+17)}{7(16 \alpha+7)}, \\
\tilde{U}^{M}=\frac{16 a^{2}(\alpha+1)^{2}}{7(16 \alpha+7)}, \\
\tilde{\pi}_{1}^{M}=\frac{216 a^{2}(\alpha+1)^{2}}{49(16 \alpha+7)^{2}}, \\
\tilde{T}^{M}=\frac{32 a^{2}(\alpha+1)(8 \alpha-1)}{7(16 \alpha+7)^{2}}, \\
\tilde{W}^{M}=\frac{144 a^{2}(\alpha+1)^{2}}{7(16 \alpha+7)^{2}} .
\end{gathered}
$$

Também neste caso, as quantidades produzidas por cada uma das empresas, e portanto a quantidade agregada no mercado, diminuem à medida que aumenta a preferência do governo pela receita fiscal.

\section{EMPRESA PRIVADA LÍDER VERSUS EMPRESA PÚBLICA LÍDER}

Nesta secção, vamos comparar os resultados obtidos num mercado em que a empresa líder é a empresa privada com os resultados obtidos num mercado em que a empresa líder é a empresa pública. Dessa comparação, resulta o seguinte teorema:

Teorema 1. Em equilíbrio, tem-se:

$$
\begin{gathered}
t^{M}>\tilde{t}^{M}, \\
q_{1}^{M}>\tilde{q}_{1}^{M}, \\
q_{2}^{M}>\tilde{q}_{2}^{M}, \text { se } \alpha>5 / 16, \\
q_{2}^{M}<\tilde{q}_{2}^{M}, \text { se } \alpha<5 / 16, \\
Q^{M}>\tilde{Q}^{M}, \\
p^{M}<\tilde{p}^{M}, \\
U^{M}>\tilde{U}^{M}, \\
\pi_{1}^{M}<\tilde{\pi}_{1}^{M}, \\
W^{M}<\tilde{W}^{M}, \\
T^{M}>\tilde{T}^{M} .
\end{gathered}
$$

Do teorema acima, concluímos que, quando a empresa líder é a empresa privada, é maior: (i) a taxa de imposto fixada pelo governo; (ii) a quantidade agregada no mercado; e (iii) a receita fiscal arrecadada pelo governo. Por outro lado, quando a empresa líder é a empresa pública, é 
maior: (i) o preço do produto em comercialização; (ii) o bem-estar social; e (iii) o lucro da empresa privada.

\section{DUOPÓLIO PÓS-PRIVATIZAÇÃO}

O duopólio pós-privatização foi também estudado por Ferreira e Ferreira (2014). Neste caso, ambas as empresas consideram os seus lucros como função objetivo. Os resultados obtidos são os seguintes:

No duopólio pós-privatização, os valores em equilíbrio são:

$$
\begin{gathered}
q_{1}^{P}=\frac{33 a(\alpha+1)}{7(33 \alpha+13)}, \\
q_{2}^{P}=\frac{55 a(\alpha+1)}{14(33 \alpha+13)}, \\
Q^{P}=\frac{121 a(\alpha+1)}{14(33 \alpha+13)}, \\
U^{P}=\frac{121 a^{2}(\alpha+1)^{2}}{28(33 \alpha+13)} \\
\pi_{1}^{P}=\frac{363 a^{2}(\alpha+1)^{2}}{14(33 \alpha+13)^{2}}, \\
\pi_{2}^{P}=\frac{9075 a^{2}(\alpha+1)^{2}}{392(33 \alpha+13)^{2}} .
\end{gathered}
$$

Vejamos, agora, o efeito da privatização no nosso modelo (empresa privada atuando como líder). Dos resultados acima, resulta que

$$
\begin{aligned}
& U^{P}<U^{M}, \text { se } \alpha>24 / 935, \\
& U^{P}>U^{M}, \text { se } \alpha<24 / 935 .
\end{aligned}
$$

Assim, se o governo tiver uma preferência pela receita fiscal suficientemente maior do que pelo bem-estar social, então a empresa pública não será privatizada; se, pelo contrário, essa preferência é apenas ligeiramente superior, então o governo tem interesse na privatização.

\section{CONCLUSÕES}

Neste trabalho, analisamos os efeitos da privatização e da preferência do governo pela receita fiscal num mercado em que as empresas competem de acordo com o modelo misto de Stackelberg, onde a empresa líder é a empresa privada. Qualitativamente, os resultados obtidos são análogos aos resultados para o modelo em que a empresa líder é a empresa pública: (i) se o governo tiver uma preferência pela receita fiscal suficientemente maior do que pelo bem-estar social, então a empresa pública não será privatizada; (ii) se, pelo contrário, essa preferência é apenas ligeiramente superior, então há interesse na privatização. Além disso, comparamos os resultados dos dois modelos, destacando-se as seguintes conclusões: (i) a taxa de imposto fixada pelo governo, a receita fiscal e a quantidade agregada no mercado são maiores quando a empresa líder é a empresa privada; (ii) o preço do produto 
em comercialização, o bem-estar social e o lucro da empresa privada são maiores quando a empresa líder é a empresa pública.

Os resultados apresentados permitem ajudar a entender as decisões que os governos tomam sobre a privatização de empresas públicas.

Agradecimentos. Agradecemos os apoios financeiros atribuídos pela ESEIG e pelo IPP.

\section{REFERÊNCIAS BIBLIOGRÁFICAS}

[1] J.A. Brander and B.J. Spencer. Tariff protection and imperfect competition. In H. Kierzkowski (ed.): Monopolistic Competition and International Trade. Oxford University Press, Oxford: 194-207, 1984.

[2] C.-C. Chao and E. Yu. Partial privatization, foreign competition, and optimum tariff. Review of International Economics, 14: 87-92, 2006.

[3] R. Clarke and D.R. Collie. Optimum-welfare and maximum-revenue tariffs under Bertrand duopoly. Scottish Journal of Political Economy, 53: 398-408, 2006.

[4] G. De Fraja and F. Delbono. Game theoretic models of mixed oligopoly. Journal of Economic Survey, 4: 1-17, 1990.

[5] F.A. Ferreira and F. Ferreira. Privatization and government preferences in a mixed duopoly: Stackelberg versus Cournot. In J.A. Tenreiro Machado et al. (eds.) Discontinuity and Complexity in Nonlinear Physical Systems. Springer International Publishing Switzerland: 421-430, 2014.

[6] F.A. Ferreira and F. Ferreira. Maximum-revenue tariff under Bertrand duopoly with unknown costs. Communications in Nonlinear Science and Numerical Simulation, 14: 3498-3502, 2009.

[7] K. Fjell and J. Heywood. Mixed oligopoly, subsidization and the order of firms's moves: the relevance of privatization. Economics Letters, 53: 411-416, 2004.

[8] H. Kato. Privatization and government preference. Economics Bulletin, 12: 1-7, 2008.

[9] B. Larue B and J.-P. Gervais. Welfare-maximizing and revenue-maximizing tariffs with few domestic firms. Canadian Journal of Economics, 35: 786-804, 2002.

[10] T. Matsumura. Partial privatization in mixed Duopoly. Journal of Public Economics, 70: 473-483, 1998.

[11] M. White. Mixed oligopoly, privatization and subsidization. Economics Letters, 53: 189-195, 1996. 